basophilic areas of calcification surrounded by granulation tissue and fibrous trabeculae. There was a noticeable inflammatory reaction, with many foreign body type giant cells. The features were characteristic of tumoral calcinosis.

After operation the wound healed well and there was no recurrence after 12 months. Serum calcium, phosphorus, and alkaline phosphatase estimations made after the tissue diagnosis were normal. It was not possible to examine relatives of this patient.

\section{Discussion}

Tumoral calcinosis is an uncommon disease which occurs predominantly in coloured races but with occasional reports in white patients. It occurs equally in males and females and at all ages. It may be that its apparent rarity is due to lack of recognition. McClatchie and Bremner (1969) stressed that cases of tumoral calcinosis masquerade under various pseudonyms such as calcified bursa, calcified lipoma, and metastatic calcification. There is a distinct clinical picture, however, and the histology is characteristic if not diagnostic. In addition, familial cases and biochemical abnormalities in patients and their asymptomatic relatives point to tumoral calcinosis as a distinct entity.

Tumoral calcinosis presents as swelling about joints. The swelling may be solitary and slow growing or multiple with rapid growth. The commonest sites for the tumour are about the hip, elbow, shoulder, and the gluteal muscles, but may occur in many other sites. The swellings occur in the subcutaneous tissues but may also be deep in the muscle planes. The masses are of variable size and hard on palpation but may occasionally feel cystic. Sinus formation is uncommon but when it occurs may be associated with discharge of white chalk-like debris. The masses are not usually accompanied by pain or tenderness but may cause such symptoms by pressure on local nerves (Riemenschneider and Ecker, 1952).

Radiologically, calcification is universal, showing initially as small discrete calcified nodules progressing to large lobulated calcified tumours. They do not affect the adjacent joint (Palmer, 1969).

The pathological appearance of these lesions is of a septate cystic mass, the septae formed by thick bands of fibrous and granulation tissue, with the intervening cisterns full of a semisolid, chalk-like material with a granular structureless appearance and which stains positively for calcium. Chemical analysis of this material in case 1 has shown it to consist of calcium phosphate. Other analyses have shown calcium phosphate alone, or a mixture of calcium phosphate and carbonate (Lafferty et al., 1969).

The serum biochemistry in tumoral calcinosis may be ab- normal as in case 1. Lafferty et al., in their review of 18 previously recorded cases, pointed out that while serum calcium and alkaline phosphatase levels were normal the serum phosphorus was increased in five of 13 patients in whom these estimations were made. Moreover, it is interesting to note that the disease may be familial with clinical lesions presenting in sibs, or healthy sibs may have only biochemical abnormalities.

Nothing definite is known of the aetiology of tumoral calcinosis. The geographical distribution and subcutaneous calcification have suggested a reaction to parasites, particularly onchocerca, but the widespread occurrence of the lesion in areas where simulium is absent rule this out (McClatchie and Bremner, 1969). Moreover, no parasitic remnants have been described in association with lesions showing the characteristic histology.

Trauma has been alleged to be a causal factor, and predominance about hips and buttocks has been ascribed to sleeping on the floor or hard beds. Thomson and Tanner (1949) described a case of a Negro "bronchobuster" who had received a kick from a horse and whose two brothers had previously been treated for the disease. Owor (1972), however, stresses that in many of the Ugandan cases the lesions were situated in sites where trauma is minimal.

The frequent raised levels of serum phosphorus and the occurrence of the disease or serum abnormalities among sibs suggest that tumoral calcinosis may be an inborn error of metabolism. Lafferty et al. (1965) made calcium, nitrogen, and phosphorus balance studies in one case but were unable to specify an exact abnormality. It is interesting to speculate that a metabolic variation, perhaps with increased frequency in the coloured races, might predispose to the development of lesions at sites of repeated trauma.

We wish to thank Mr. A. G. Cox for clinical details of Case 2 and Mr. G. Padmore for the biochemical results. We thank Miss B. Pentland for her typing.

\section{References}

Berg, B. (1972). British fournal of Surgery, 59, 570. Inclan, A. (1943). Fournal of the American Medical Association, 121, 490. Lafferty, F. W., Reynolds, E. S., and Pearson, O. H. (1965). American Fournal of Medicine, 38, 105.

McClatchie, S., and Bremner, A. D. (1969). British Medical fournal, 1, 153.

Maegraith, B. (1971). Practitioner, 206, 816

Owor, R. (1972). Tropical and Geographical Medicine, 24, 39.

Palmer, P. E. S. (1966). British fournal of Radiology, 31, 104

Palmer, P. E. S. (1969). Quoted by McClatchie and Bremner (1969).

Riemenschneider, P. A., and Ecker, A. (1952). Fournal of Neurosurgery, 9 304.

Thomson, J. E. M., and Tanner, F. H. (1949). fournal of Bone and foint Surgery, 31A, 132.

\title{
Enzyme Typing of Malaria Parasites
}

\section{R. CARTER, A. VOLLER}

British Medical fournal, 1973, 1, 149-150

\section{Summary}

Analysis of various isolates of Plasmodium falciparum from East and West Africa and from South-east Asia showed that some of the parasite enzymes can exist in

Protozoan Genetics Unit, Institute of Animal Genetics, Edinburgh R. CARTER, PH.D., Research Associate

Nuffield Institute of Comparative Medicine, Zoological Society of London, London N.W.1

A. VOLLER, D.sc., Senior Lecturer more than one electrophoretic form. At least one form of each enzyme was common to parasites from all three regions. The enzyme forms could be used to differentiate morphologically indistinguishable samples of $P$. falciparum.

\section{Introduction}

Malignant tertian malaria, caused by infection of man by Plasmodium falciparum, is widely distributed throughout the tropical areas of the world. In spite of the fact that "strains" isolated from within and between different geographical regions differ in their immunological characteristics, in drug sensitivity, 
and in transmissibility (Garnham, 1966), all are grouped on morphological criteria within the single species $P$. falciparum. If we are to understand the mechanisms whereby individuals develop immunity to malaria under natural conditions, and to learn how drug resistant malaria can spread, it is important that we should be able to identify and characterize such distinct parasite populations that may exist. As recent immunological tests have not provided information of this kind, and in view of Carter's (1970) differentiation of distinct groups of rodent malaria parasites on the basis of enzyme variants, we thought it worthwhile to see if the techniques could be applied to human malaria.

\section{Materials and Methods}

Three "strains" of $P$. falciparum were used: (1) West Africanoriginated in Lagos, West Africa; (2) Palo Alto-originated in Uganda, East Africa; and (3) Camp-originated in South-east Asia.

These strains had been serially passaged by blood transfer between Aotus trivirgatus (owl monkeys) for several years.

For the present analyses blood was obtained from acutely infected aotus monkeys when more than $10 \%$ of the erythrocytes contained maturing malaria parasites.

Two additional blood samples from human placentae obtained from Gambians with naturally acquired $P$. falciparum infections were donated by Dr. R. J. M. Wilson (National Institute for Medical Research).

In all cases whole parasitized blood was used. This was stored as freeze-dried material and was reconstituted in a minimum volume of distilled water for electrophoresis. Uninfected blood treated in the same way was used as control material to show the position of the host enzymes after electrophoresis.

\section{ELECTROPHORESIS METHODS}

The technique of horizontal starch gel electrophoresis was used. TRIS citrate buffer pH 6.0 was used for electrophoresis of lactate dehydrogenase, 6-phosphogluconate dehydrogenase, and glucose phosphate isomerase. Electrophoresis of the latter enzyme was also carried out in TRIS-HCl buffer pH 8.0.

After electrophoresis the positions of the enzyme bands were made visible by linking the enzyme reactions to the deposition of the insoluble blue dye MTT-tetrazolium, as described by Carter (1970).

\section{Results}

Malaria parasite enzymes which could be differentiated from those of the host were identified in all the samples tested. A typical electrophoretic run of glucose phosphate isomerase is shown in the fig.

A single band of parasite 6-phosphogluconate was found in all the samples. Two forms of lactate dehydrogenase and glucose phosphate isomerase were identified. Only when the electrophoresis was carried out in the TRIS- $\mathrm{HCl}$ pH 8.0 buffer was the glucose phosphate isomerase separable into the two forms.

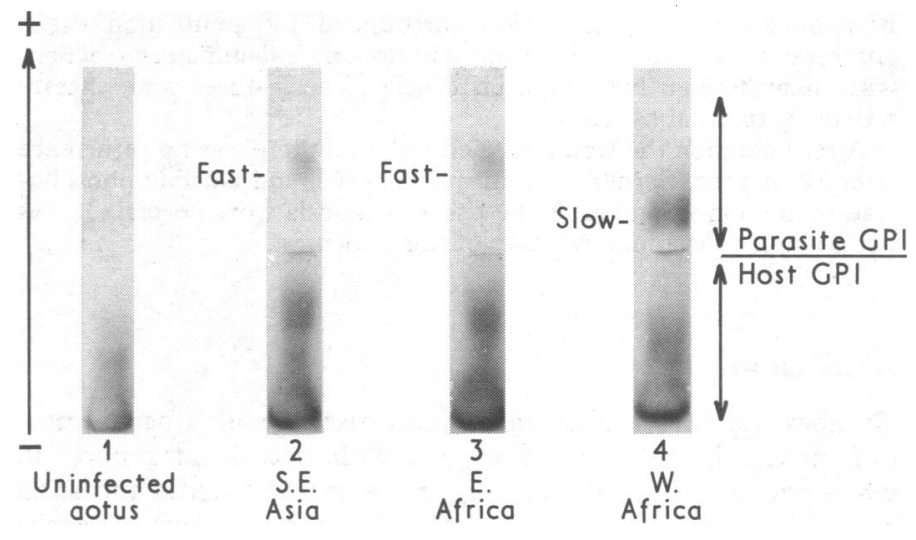

Starch gel electrophoresis in TRIS-HCl buffer $\mathrm{pH} 8.0$ of glucose phosphate isomerase comparing (1) uninfected aotus blood with blood samples from aotus monkeys infected with isolates of $P$. falciparum from (2) S. E. Asia, (3) East Africa, and (4) West Africa.

The East African and South-east Asian isolates of $P$. falciparum showed only the fast form of glucose phosphate isomerase whereas the West African isolate showed only the slow moving form. Both placental samples from West Africa showed both forms. The fast moving form of lactate dehydrogenase was found in all samples, while the slow moving form occurred in only one of the placental samples from West Africa.

\section{Discussion}

These results show that morphologically indistinguishable isolates of $P$. falciparum can, in some instances, be separated on the basis of particular enzyme variants.

These preliminary data show that $P$. falciparum parasites include genetically polymorphic types. However, the parasites from West Africa, East Africa, and South-east Asia cannot be placed in separate geographical groups on the basis of the enzyme analyses since, for each enzyme, the same form can be found in material from each region. As the East African and South-east Asian parasite samples were represented by single isolates we cannot, at present, say whether the polymorphism found in samples from West Africa extends to the parasites from the other regions. Field studies designed to assess the incidence of the polymorphic enzyme variants and their relation to antigenic types of $P$. falciparum are now in progress.

This preliminary work indicates that enzyme characterization can be successfully applied to human malaria, and suggests that it may be of value in unravelling the outstanding epidemiological questions related to the existence of distinct but morphologically identical malaria parasite populations.

We are grateful for the financial support from the World Health Organization and the Medical Research Council of Great Britain.

\section{References}

Carter, R. (1970). Transaction of the Royal Society of Tropical Medicine and

Hygiene, 64, 401. (1966). Malaria Parasites and Other Haemosporidia, Oxford, Blackwell Scientific. 